\title{
Validação de escala diagramática para quantificação da severidade da antracnose do colmo do milho
}

\author{
Diagrammatic scale validation to quantify the severity of \\ anthracnose stalk in corn
}

\author{
Alessandro Nicoli ${ }^{\mathrm{I}}$ Rodrigo Veras da Costa ${ }^{\mathrm{I}{ }^{*}}$ Luciano Viana Cota ${ }^{\mathrm{II}}$ \\ Dagma Dionísia da Silva ${ }^{I I}$ Laércio Zambolim ${ }^{I}$ Fabrício Esutáquio Lanza \\ Daniel Pereira Guimarães ${ }^{I I}$ Elena Charlotte Landau ${ }^{I I}$
}

RESUMO

\begin{abstract}
Objetivou-se propor e validar uma escala diagramática para quantificar a antracnose do colmo em milho. A severidade da antracnose foi estimada por dez avaliadores sem o uso de escala em 139 entrenós, e os mesmos avaliadores avaliaram os entrenós usando uma escala publicada anteriormente na literatura (primeira escala) e uma nova escala proposta. Com o uso da escala proposta, todas as variáveis foram significativamente diferentes em relação à primeira escala, e os valores foram mais próximos de uma medição acurada ( $r=0,97, C_{b}=0.98, u=0.09$, $\left.v=1.06, \rho_{c}=0.96\right)$. Os maiores desvios do erro foram observados quando os avaliadores atribuiram notas sem o uso de escalas e com a primeira escala, com vários exemplos de erros maiores do que $30 \%$ do valor real. Com a escala proposta, poucos erros acima de $15 \%$ do valor real foram encontrados, e a maioria das notas dos avaliadores não ultrapassou, para mais ou para menos, $10 \%$ do valor real. Uma maior confiabilidade na estimativa da severidade da doença foi obtida com o uso da escala proposta, com a qual $100 \%$ das comparações resultaram em um $R^{2}>0,90$. A nova escala diagramática proposta no presente trabalho melhorou a acurácia, precisão e confiabilidade das estimativas da severidade da antracnose do colmo em milho.
\end{abstract}

Palavras-chave: Zea mays, Colletotrichum graminicola, podridão colmo, quantificação.

\section{ABSTRACT}

This study aimed to validate a new diagrammatic scale to anthracnose stalk in corn. Anthracnose severity was estimated by ten raters without and with use of new scale on 139 internodes with different severity degrees and all evaluators rated the same internodes using the old scale published in the literature and a new proposed scale. All variables of the propose scale, were significantly different than those of old scale, and the values were closer to an accurate measurement ( $r=0.97, C b=0.98, u=0: 09$, $v=1: 06, \rho c=0.96)$. The large error deviations were observed when evaluators given notes without the use of scales and also with the old scale, for examples with errors up to $30 \%$. On the other hand the majority errors with the use the new scale were lower than $10 \%$. Higher reliability was achieved with the use of the proposed scale, where $100 \%$ of the comparisons were achieved $R 2>0.90$. According to the results the diagrammatic scale proposed in this study improves accuracy, precision and reliability of the evaluators to estimates the anthracnose stalk severity in corn.

Key words: Zea mays, Colletotrichum graminicola, stalk rot, quantification.

\section{INTRODUÇÃO}

A antracnose do colmo Colletotrichum graminicola (Ces.) Wils é uma das doenças mais importantes na cultura do milho em vários países, causando acamamento das plantas, redução na produção e qualidade dos grãos (BERGSTROM \& NICHOLSON, 1999; COTA et al., 2012; MATIELLO et al., 2013). Os sintomas da antracnose do colmo são mais visíveis após o florescimento das plantas de milho, porém o processo infeccioso inicia-se pelos propágulos provenientes das lesões foliares ou daqueles presentes em restos culturais. $\mathrm{Na}$ casca, surgem lesões estreitras, longitudinais e com aspecto encharcado, que são inicialmente de coloração pardo-avermelhada, posteriormente tornando-se marromescuras a negras. Nos tecidos internos do colmo, ocorre uma coloração marrom-escura, que entra em processo de desintegração, afetando o fluxo de água e nutrientes do colmo para a parte superior, podendo ocasionar a morte prematura e o acamamento das plantas (KELLER \&

IDepartamento de Fitopatologia, Universidade Federal de Viçosa (UFV), Viçosa, MG, Brasil.

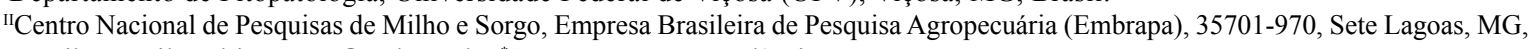
Brasil. E-mail: rodrigo.veras@embrapa.br. *Autor para correspondência. 
BERGSTROM, 1988; BERGSTROM \& NICHOLSON, 1999; VENARD \& VAILLANCOURT, 2007).

A estimativa da intensidade da antracnose do colmo é importante para avaliar as medidas de controle, resistência varietal, eficiência de fungicidas, a relação com os danos de produção, etc. Com isso, as características de acuracidade, precisão e reprodutibilidade são muito desejáveis na avaliação. No milho, a antracnose do colmo é avaliada por meio de uma escala de notas que possui classes de intensidade da doença seguindo uma progressão aritmética, mas com grandes intervalos em severidade entre as notas, aqui chamada de primeira escala (CHRISTENSEN \& WILCOXSON, 1966; ANDERSON \& WHITE, 1994; TODD \& KOMMEDAHL, 1994; AFOLABI et al., 2008). Alguns pesquisadores utilizam escala com intervalos menores nas primeiras notas $\mathrm{e}$ maiores nas seguintes, além de adotar o ponto médio de severidade para realizar a análise estatística (KELLER \& BERGSTROM, 1988; MUIMBAKANKOLONGO \& BERGSTROM, 1992; MUIMBA-KANKOLONGO \& BERGSTROM, 2011). No entanto, grandes intervalos de notas, como 0 - $25 \%$ ou $25-75 \%$, podem dificultar a precisão da avaliação da doença no colmo, tanto em infecção natural quanto em inoculação artificial, em relação ao valor real da severidade, além de afetar a diferença significativa entre os tratamentos por meio das análises estatísticas. Portanto, para melhorar a acurácia e precisão da estimativa da severidade da doença, é necessário o desenvolvimento e validação de uma escala mais precisa. Considerando esses fatores, o objetivo do presente estudo foi propor e validar uma escala diagramática para a avaliação da podridão do colmo, causada por $\boldsymbol{C}$. graminicola .

\section{MATERIAL E MÉTODOS}

Para elaboração da escala, 60 plantas do híbrido simples BRS1010 (Embrapa) foram inoculadas com o fungo $\boldsymbol{C}$. graminicola em casa de vegetação. As plantas foram inoculadas na fase de pré-pendoamento (aproximadamente 60 dias após o plantio), no terceiro entrenó previamente desinfestado com álcool $70 \%$, adotando-se o método do palito imerso na suspensão de esporos $\left(10^{6}\right.$ conídios $\left.\mathrm{ml}^{-1}\right)$. Três plantas perfuradas apenas com palito estéril foram utilizadas como testemunha.

A avaliação da antracnose do colmo foi realizada aos 30 dias após a inoculação. Para isso, os 63 colmos foram cortados longitudinalmente e as lesões foram fotografadas em um total de 139 entrenós que apresentaram sintomas da doença.
As imagens foram feitas com câmera fotográfica digital, com resolução de 16.2 Megapixel. Os colmos foram colocados em uma bancada sobre uma folha de papel plastificado de dimensões conhecidas $(80 \mathrm{x}$ $80 \mathrm{~cm}$ ) e as fotos foram tomadas em posição oblíqua, em distância que permitiu o recobrimento de toda a superfície a ser imageada. A correção geométrica das imagens foi feita com o uso do programa de sistemas de informações geográficas (SIG) Quantum Gis Versão 8 - Lisboa <http://www.qgis.org>, software livre da Open Source Geospatial Foundation OSGeo, através do módulo de georreferenciamento de imagens, tendo como pontos de controle as dimensões da folha usada como referencial. O reconhecimento de padrões de infecção, conversão de área da imagem em área real, estabelecendo o percentual de área necrosada (severidade real), foi realizado utilizando o software livre Hypercube, desenvolvido pelo Centro de Engenharia Topográfica das Forças Armadas Americanas <http://www.agc.army.mil/hypercube $>$.

Para a validação da escala proposta, as 139 imagens de entrenós de colmo contendo os diferentes graus de severidade real da antracnose do colmo foram apresentadas para dez avaliadores, os quais analisaram a severidade da doença, sem e com o uso de duas escalas, uma chamada de primeira escala e a escala proposta no presente trabalho. Primeira escala: 1 (0$25 \%), 2(26-50), 3(51-75 \%)$ e $4(76-100 \%)$ e escala proposta: 1 (0-12,5\%), 2 (12,6-25\%), 3 (25,1-37,5\%), $4(37,6-50 \%), 5(50,1-62,5 \%), 6(62,6-75 \%), 7$ (75,1$87,5)$ e $8(87,6-100)$. Para cada imagem avaliada, o valor sem escala foi de 0 a 100, e com o uso das escalas o valor adotado foi referente ao ponto médio. Por exemplo, para a nota 1 , com a primeira escala, o ponto médio é $12,5 \%$ e, para a escala proposta, é 6,25\%.

Após a obtenção dos dados de cada avaliador, esses foram submetidos a uma análise de regressão linear simples entre a severidade real (variável independente) e a severidade estimada (variável dependente), sem e com a utilização das duas escalas. Com isso, foi obtido o coeficiente de determinação $\left(\mathrm{R}^{2}\right)$ e a variância dos erros (valor estimado menos a severidade real) para cada avaliador. A reprodutibilidade das avaliações ou confiança entre os avaliadores foi determinada com base nos valores de $\mathrm{R}^{2}$ da regressão linear entre as severidades estimadas pelos 10 diferentes avaliadores combinados aos pares, como proposto por NUTTER \& SCHULTZ (1995), sendo um total de 45 combinações. As análises de regressão entre a severidade real e a estimada para cada avaliador, bem como entre os avaliadores, foram realizadas por meio do programa estatístico MINITAB, versão 14. 
Além da regressão linear entre severidade real e estimada, a acurácia e a precisão também foram determinadas, usando a análise de correlação concordante de Lin $\left(\rho_{c}\right)$, a qual combina as medidas de acurácia e precisão para avaliar o grau em que os pares das observações se deslocam em relação à linha concordante de $45^{\circ}$ (com intercepto $=0$ e inclinação $=1$ ). A fórmula utilizada para o $p c$ foi a seguinte: $p c=C b . r$, em que $C b$ é um fator de correção do desvio que mede o quão longe está a linha ajustada dos desvios em relação à linha concordante e, portanto, é uma medida de acurácia, e $r$, que é o coeficiente de correlação entre a severidade estimada (Y) e a severidade real $(\mathrm{X})$, sendo uma medida de precisão. $C b$ é derivado de: $C_{b}=\left[\left(v+1 / v+u^{2}\right) / 2\right]^{-1}$, onde $v=\sigma_{y} / \sigma_{x}$, em que $\sigma$ l o desvio-padrão de $Y$ e $X$, respectivamente; e $u=\left(\mu_{\mathrm{y}}-\mu_{\mathrm{x}}\right) /\left(\sigma_{\mathrm{y}} \cdot \sigma_{\mathrm{x}}\right)^{-1 / 2}$, onde $\mu \mathrm{\imath}$ o valor médio de $Y$ e $X$, respectivamente, $\sigma$ l definido como acima. $\mathrm{O}$ componente $v$ mede a diferença de escala entre os valores de Y e X, que, essencialmente, é definida pela diferença na inclinação das duas linhas (linha ajustada da regressão com a linha concordante). Iguais inclinações para as duas linhas implica um valor de $v$ igual a 1 . O termo $u$ reflete a mudança de localização da linha ajustada da regressão em relação à linha concordante, que é caracterizado pela diferença de altura entre essas duas linhas. Iguais alturas implicam um valor de $u$ igual a 0 . Portanto, um avaliador perfeito é aquele em que suas estimativas estão todas iguais à linha concordante, ou seja, $r=$ $1, C b=1[v=1, u=0]$, e, consequentemente, $p c=1$ (NITA et al., 2003; BOCK et al., 2010; CAPUCHO et al., 2011; YADAV et al., 2012).

Para todas essas variáveis obtidas pela análise de correlação concordante de Lin, incluindo a reprodutibilidade, a diferença entre a média foi calculada (com escala - sem escala e escala proposta - primeira escala) e um teste seguinte foi usado para analisar sua significância (Tabela 1) (YI et al., 2008; BARDSLEY \& NGUGI, 2012; YADAV et al., 2012;

Tabela 1 - Efeito da escala proposta na acurácia e precisão, por meio da avaliação da antracnose do colmo em 139 entrenós por $1 C$ avaliadores.

\begin{tabular}{|c|c|c|c|c|}
\hline \multirow{2}{*}{ Variável $^{\mathrm{a}}$} & - & 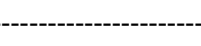 & \multirow{2}{*}{$\mathrm{DM}^{\mathrm{c}}$} & \multirow{2}{*}{ IC $95 \%{ }^{\mathrm{d}}$} \\
\hline & Sem escala & Primeira escala & & \\
\hline$r$ & $0,91(0,06)$ & $0,90(0,06)$ & $-0,011(0,0001)$ & $-0,024-0,003^{\mathrm{ns}}$ \\
\hline$C_{b}$ & $0,90(0,07)$ & $0,94(0,03)$ & $0,035(0,0004)$ & $-0,002-0,077^{\mathrm{ns}}$ \\
\hline$u$ & $0,40(0,19)$ & $0,32(0,16)$ & $-0,076(0,0013)$ & $-0,192-0,039^{\mathrm{ns}}$ \\
\hline$v$ & $1,12(0,12)$ & $1,00(0,02)$ & $-0,118(0,0009)$ & $-0,192--0,0315^{*}$ \\
\hline$\rho_{c}$ & $0,82(0,07)$ & $0,84(0,05)$ & $0,018(0,0003)$ & $-0,013-0,054^{\mathrm{ns}}$ \\
\hline Variável & Sem escala & Escala proposta & DM & IC 95\% \\
\hline$r$ & $0,91(0,06)$ & $0,97(0,02)$ & $0,060(0,0003)$ & $0,034-0,089^{*}$ \\
\hline$C_{b}$ & $0,90(0,07)$ & $0,98(0,01)$ & $0,083(0,0004)$ & $0,048-0,125^{*}$ \\
\hline$u$ & $0,40(0,19)$ & $0,09(0,07)$ & $-0,306(0,0010)$ & $-0,404--0,225^{*}$ \\
\hline$v$ & $1,12(0,12)$ & $1,06(0,03)$ & $-0,063(0,0007)$ & $-0,126-0,007^{\mathrm{ns}}$ \\
\hline$\rho_{c}$ & $0,82(0,07)$ & $0,96(0,02)$ & $0,134(0,0004)$ & $0,097-0,172^{*}$ \\
\hline Variável & Primeira escala & Escala proposta & DM & IC 95\% \\
\hline$R$ & $0,90(0,06)$ & $0,97(0,02)$ & $0,071(0,0002)$ & $0,049-0,10^{*}$ \\
\hline$C_{b}$ & $0,94(0,03)$ & $0,98(0,01)$ & $0,047(0,0001)$ & $0,030-0,062^{*}$ \\
\hline$U$ & $0,32(0,16)$ & $0,09(0,07)$ & $-0,229(0,0009)$ & $-0,308--0,139^{*}$ \\
\hline$Y$ & $1,00(0,02)$ & $1,06(0,03)$ & $0,055(0,0002)$ & $0,031-0,077^{*}$ \\
\hline$\rho_{c}$ & $0,84(0,05)$ & $0,96(0,02)$ & $0,115(0,0002)$ & $0,090-0,139^{*}$ \\
\hline
\end{tabular}

${ }^{\mathrm{a}} r$ : coeficiente de correlação entre severidade estimada e a real (medida de precisão); $C b$ : fator de correção do desvio que mede o quão long€ está a linha ajustada dos desvios em relação à linha concordante (medida de acurácia); $u$ : diferença de altura entre a linha da regressão e a linha concordante; $v$ : diferença na inclinação das duas linhas (regressão e concordante); $\rho_{c}$ : coeficiente de correlação concordante de Lin.

${ }^{\mathrm{b}}$ Desvio padrão em parênteses.

${ }^{c}$ Diferença média (DM) entre cada avaliação, com erro padrão em parênteses

${ }^{\mathrm{d}}$ Intervalo de confiança (IC). Incluindo o zero, a diferença não é significativa $(\mathrm{P}=0,05) ;{ }^{\text {ns }}$ não significativo, ${ }^{*}$ significativo.

Ciência Rural, v.45, n.10, out, 2015. 
RIOS et al., 2013). O teste de equivalência foi usado para calcular o intervalo de confiança (IC) a $95 \%$ de probabilidade para cada variável por bootstrapping, usando o método do percentil. Se o IC para uma determinada variável não incluiu o valor zero, indica que a diferença é significativa $(\mathrm{P}=0,05)$, ou seja, provase que o uso da escala melhorou significativamente a variável em análise, baseado-se nas estimativas de todos avaliadores.

\section{RESULTADOS}

A escala diagramática proposta neste trabalho contém oito classes de notas de intensidade da antracnose do colmo (1=6,25\%; 2=18,8\%; $3=31,3 \% ; 4=43,8 \% ; 5=56,3 \% ; 6=68,8 \% ; 7=81,3 \%$; $8=93,8 \%$ ), seguindo uma progressão aritmética e com intervalos menores de severidade entre as notas, a qual pode ser observada na figura 1 .

Baseado na análise de correlação concordante de Lin e teste de equivalência, além da linha de regressão obtida entre a severidade real e estimada, as avaliações realizadas por todos os avaliadores foram mais próximas ao valor real com o uso da escala proposta, quando comparada com a primeira escala ou com as avaliações sem o auxílio de escala (Tabela 1 e 2). De fato, as medidas de acurácia e precisão melhoraram significativamente com o resultado da utilização da escala proposta para avaliar a severidade da antracnose do colmo no milho.

Ao se analisar a primeira escala, apenas a variável $v$ foi significativa em relação às avaliações sem escala, no entanto, as outras variáveis apresentaram uma pequena diferença numérica e não foram significativamente diferentes. Entretanto, com o uso da escala proposta, o $v$ não foi significativo em relação à sem escala, mas todas as outras variáveis foram significativas. Por meio do uso dessa escala, todas as variáveis foram significativamente diferentes em relação à primeira escala, e os valores apresentados foram mais próximos de uma medição acurada $\left(r=0.97, C_{b}=0.98, u=0.09, v=1.06, \rho_{c}=0.96\right)$ (Tabela 1). Além disso, para $100 \%$ dos avaliadores, o coeficiente da análise de correlação concordante de Lin $\left(\rho_{c}\right)$ foi maior com o uso da escala proposta.

Com o uso da escala proposta, foi observado um aumento da precisão para todos os avaliadores, pois os níveis de precisão das estimativas visuais da antracnose do colmo apresentaram valores de coeficiente de determinação $\left(\mathrm{R}^{2}\right)$ superiores a $95 \%$

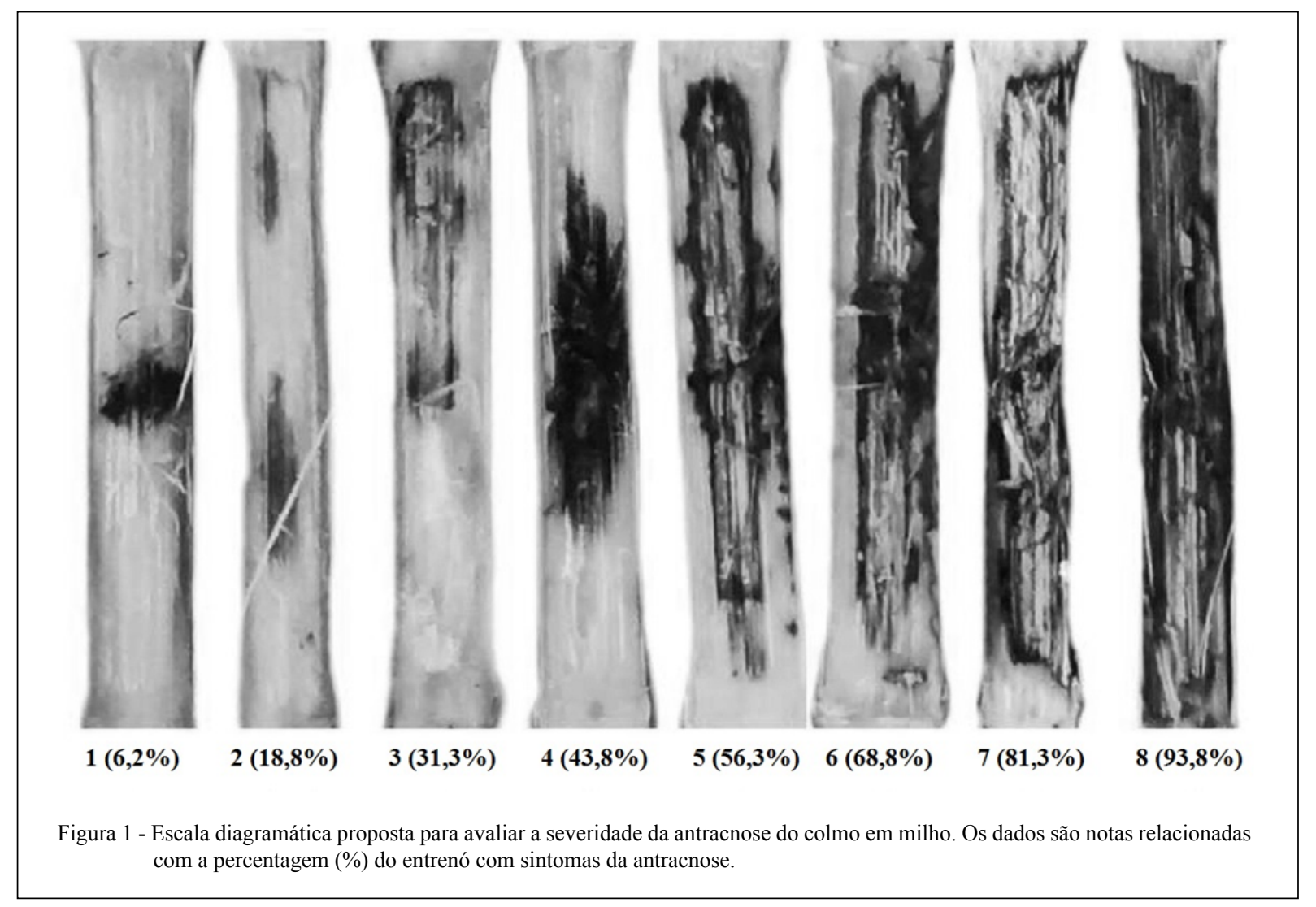

Ciência Rural, v.45, n.10, out, 2015. 
Tabela 2 - Reprodutibilidade da avaliação visual em 139 entrenós com antracnose do colmo por 10 avaliadores, sem e com o uso das duas escalas. Reprodutibilidade é medida por coeficiente de determinação $\left(\mathrm{R}^{2}\right)$.

\begin{tabular}{lcc}
\hline Estatística & Sem escala & Primeira escala \\
\hline Coeficiente de determinação médio entre avaliadores $\left(\mathrm{R}^{2}\right)^{\mathrm{a}}$ & $0,83(0,55-0,95)$ & $0,83(0,64-0,93)$ \\
Diferença média (primeira escala - sem escala) & $0,0026(0,0002)-(\mathrm{IC}-0,0159-0,0216)^{\mathrm{ns}}$ \\
Diferença média (escala proposta - sem escala) & $0,1136(0.0003)-(\mathrm{IC}-0,0813-0,1482)^{*}$ \\
Diferença média (escala proposta - primeira escala) & $0,1133(0,0002)-(\mathrm{IC}-0,0938-0,1356)^{*}$ \\
\hline
\end{tabular}

${ }^{\mathrm{a} C}$ Coeficiente médio de determinação estimado das comparações aos pares da avaliação por todos avaliadores.

${ }^{b}$ Média da diferença entre cada avaliação, com erro padrão em parênteses, intervalo de confiança (IC 95\%) baseado em bootstrap. Se no IC incluiu zero, a diferença não é significativa $(\mathrm{P}=0,05) .{ }^{\text {ns }}$ não significativo, ${ }^{*}$ significativo.

(dados não mostrados), podendo ser considerados altos para este tipo de avaliação. Além disso, uma melhor precisão também foi confirmada pela diminuição dos erros absolutos (Tabela 1). Sem a utilização de escala ou com o uso da primeira escala, os avaliadores apresentaram mais desvios em erros e, com exceção do avaliador 1, todos os outros avaliadores apresentaram erros mais alto que $20 \%$, com vários exemplos de erros maiores do que $30 \%$. Entretanto, com o uso da escala proposta, poucos erros foram acima de $15 \%$, e a maioria foi concentrada no intervalo de $10 \%$.

A reprodutibilidade foi melhorada significativamente por meio da utilização da escala proposta (Tabela 2). Sem o uso de escala e com a primeira escala, $60 \%$ e $93 \%$ das comparações ao pares mostraram um $\mathrm{R}^{2}<0,89$, respectivamente. Enquanto isso, com a escala proposta, $100 \%$ das comparações resultaram em um $\mathrm{R}^{2}>0.90$, indicando assim resultados confiáveis. Enquanto o coeficiente de determinação médio entre avaliadores $\left(\mathrm{R}^{2}\right)$ foi de $83 \%$ para o uso sem escala e com primeira escala, com a escala proposta a média foi de $95 \%$, com pouca variação. Além disso, a análise de diferença média (bootstrapping) demonstrou um efeito significativo da escala proposta, em que os avaliadores podem avaliar a doença de maneira mais uniforme (Tabela 2).

\section{DISCUSSÃO}

A escala diagramática proposta teve uma boa representação dos sintomas da antracnose do colmo, melhorando a habilidade dos avaliadores para avaliar a severidade de forma mais acurada e precisa nos 139 entrenós apresentados neste trabalho. $\mathrm{Na}$ avaliação de um experimento futuro, a análise estatística provavelmente será mais significativa e os tratamentos serão mais diferenciados com o uso da escala proposta, pois o intervalo em severidade entre as notas é menor em comparação ao uso da primeira escala. Portanto, os resultados aqui obtidos reforçam a importância de validação de uma escala para as futuras avaliações de doenças em plantas, conforme encontrado em vários outros estudos (STONEHOUSE, 1994; LEITE \& AMORIN, 2002; GOMES et al., 2004; MICHEREFF et al., 2006; CAPUCHO et al., 2010; CAPUCHO et al., 2011; YADAV et al., 2012, RIOS et al., 2013).

Todas as análises utilizadas demonstraram que a escala proposta melhorou de forma significativa a estimativa da severidade da antracnose do colmo, comparada ao não uso de escala ou a quando se utilizou a primeira escala. As avaliações realizadas por todos os avaliadores foram mais próximas ao valor real com a escala proposta. Além disso, 100\% dos avaliadores apresentaram maiores valores de coeficiente da análise de correlação concordante de Lin $\left(\rho_{c}\right)$ e as variáveis foram significativas pelo teste de equivalência quando a escala proposta foi adotada. Mesmo com a utilização da análise de regressão, o uso do $\rho_{c}$ e o teste de equivalência ( $95 \%$ IC) foram importantes para interpretação das avaliações realizadas pelos dez avaliadores. O coeficiente de Lin $\left(\rho_{c}\right)$ tem sido usado com muito sucesso em vários trabalhos (BOCK et al., 2010; CAPUCHO et al., 2011; YADAV et al., 2012; RIOS et al., 2013), e mais recentemente o teste de equivalência também, facilitando ainda mais a conclusão dos resultados (BARDSLEY \& NGUGI, 2012; YADAV et al., 2012; RIOS et al., 2013).

Os avaliadores tiveram uma tendência em superestimar a severidade da doença, a qual tem sido observada em vários outros estudos envolvendo validação de escalas (NEWTON \& HACKETT, 1994; PARKER et al., 1995; DIAZ et al., 2001; 
LEITE \& AMORIM, 2002; CAPUCHO et al., 2010; CAPUCHO et al., 2011; RIOS et al., 2013). Apenas em alguns poucos estudos tem sido verificada a severidade subestimada (MICHEREFF et al., 1998, 2000; GOMES et al., 2004).

Nas estimativas da antracnose do colmo, o erro absoluto foi reduzido usando-se a escala proposta, em que a maioria dos erros foi concentrada no intervalo de $10 \%$. Portanto, o treinamento dos avaliadores com uso dessa escala reduz os erros absolutos, aumentando a eficiência e padronizando os resultados das avaliações (NUTTER \& SCHULTZ, 1995; STONEHOUSE, 1994). A confiabilidade de estimativas entre os avaliadores também foi melhorada, resultando em $100 \%$ das comparações $\mathrm{R}^{2}>0,90$, indicando a efetividade da escala proposta. Sem o uso de escala e com a primeira escala, o coeficiente de determinação médio entre avaliadores $\left(\mathrm{R}^{2}\right)$ foi de $83 \%$, enquanto com a escala proposta a média foi de $95 \%$, com pouca variação, além de apresentar um efeito significativo pela análise de bootstrapping. Quando diferentes avaliadores usam a mesma escala para avaliar severidade de doenças, é importante que as estimativas sejam similares (NUTTER \& SCHULTZ, 1995).

Como conclusão geral, a escala proposta melhorou a acurácia, precisão e confiabilidade das estimativas da severidade da antracnose do colmo em milho. Portanto, essa escala será uma ferramenta de grande utilidade para estudos epidemiológicos, caracterização de isolados de $\boldsymbol{C}$. graminicola, danos de produção, métodos de controle da antracnose do colmo e outras pesquisas envolvendo podridão do colmo na cultura do milho.

\section{AGRADECIMENTOS}

Os autores agradecem ao Conselho Nacional de Desenvolvimento Científico e Tecnológico $(\mathrm{CNPq})$ e à Embrapa Milho e Sorgo, pelo apoio financeiro.

\section{REFERÊNCIAS}

AFOLABI, C.G et al. Novel sources of resistance to Fusarium stalk rot of maize in tropical Africa. Plant Disease, v.92, p.772780, 2008. Disponível em: <http://apsjournals.apsnet.org/ doi/pdf/10.1094/PDIS-92-5-0772>. Acesso em: 20/10/2014 doi:10.1094/ PDIS-92-5-0772.

ANDERSON, B; WHITE, D.G. Evaluation of methods for identification of corn genotypes with stalk rot and lodging resistance. Plant Disease, v.78, p.590-593, 1994. Disponível em: $<$ http://www.apsnet.org/publications/plantdisease/backissues/ Documents/1994Articles/PlantDisease78n06 590.PDF>. Acesso em: 20/10/2014. doi: 10.1094/PD-78-0590.
BARDSLEY, S.J.; NGUGI, H.K. Reliability and accuracy of visual methods used to quantify severity of foliar bacterial spot symptoms on peach and nectarine. Plant Pathology, v.61, p.460-474, 2012. Disponível em: <http://onlinelibrary.wiley.com/doi/10.1111/ j.1365-3059.2012.02651.x/epdf>. Acesso em: 20/10/2014. doi: 10.1111/j.1365-3059.2012.02651.x.

BERGSTROM, G.C.; NICHOLSON, R.L. The biology of corn anthracnose: knowledge to exploit for improved management. Phytopathology, v.83, p.596-608, 1999. Disponível em: <http:// apsjournals.apsnet.org/doi/pdf/10.1094/PDIS.1999.83.7.596>. Acesso em: 20/03/2015. doi: 10.1094/PDIS.1999.83.7.596.

BOCK, C.H. et al. Plant disease severity estimated visually, by digital photography and image analysis, and by hyperspectral imaging. Critical Reviews in Plant Sciences, v.29, p.59-107, 2010. Disponível em: $<$ http://naldc.nal.usda.gov/naldc/download. $\mathrm{xhtml}$ ?id=43374\&content $=\mathrm{PDF}>$. Acesso em: 20/03/2015. doi: $10.1080 / 07352681003617285$

CAPUCHO, A.S. et al. Influence of leaf position that correspond to whole plant severity and diagrammatic scale for white spot of corn. Crop Protection, v.29, p.1015-1020, 2010. Disponível em: <http://ac.els-cdn.com/S0261219410001079/1-s2.0S0261219410001079-main.pdf?_tid=470b7ba4-cf37-11e49402-00000aacb360\&acdnat $=1426879885 \quad 1732278778 \mathrm{bcb} 4 \mathrm{f}$ a42b2af5fb9374256>. Acesso em: 20/03/2015. doi: 10.1016/j. cropro.2010.04.012.

CAPUCHO, A.S. et al. Development and validation of a standard area diagram set to estimate severity of leaf rust in Coffea arabica and $\boldsymbol{C}$. canephora. Plant Pathology, v.60, p.1144-1150, 2011. Disponível em: <http://onlinelibrary.wiley.com/doi/10.1111/ j.1365-3059.2011.02472.x/epdf $>$. Acesso em: 20/03/2015. doi: 10.1111/j.1365-3059.2011.02472.x.

CHRISTENSEN, J.J.; WILCOXSON, R.D. Stalk rot of corn. St. Paul: American Phytopathological, (American Phytopathological Society. Monograph, 3), 1966. 59p.

COTA, L.V. et al. Quantification of yield losses due to anthracnose stalk rot on corn in Brazilian conditions. Journal of Phytopathology, v.160, p.680-684, 2012. Disponível em: $<$ http:// onlinelibrary.wiley.com/doi/10.1111/jph.12008/epdf >. Acesso em: 20/03/2015. doi: 10.1111/jph.12008

DIAZ, C.G. et al. Desenvolvimento e validação de uma escala diagramática para Xanthomonas axonopodis pv. phaseoli em feijoeiro. Summa Phytopatologica, v.27, p.35-39, 2001. Disponível em: $\quad<$ http://www.scielo.br/pdf/sp/v35n3/a12v35n3.pdf $>$. Acesso em: 20/03/2015. doi: 10.1590/S0100-54052009000300012.

GOMES, A.M.A. et al. Elaboração e validação de escala diagramática para cercosporiose da alface. Summa Phytopathologica, v.30, p.38-42, 2004. Disponível em: <https:// docs.google.com/file/d/0B6r87-taaOV7Wm1TR3JYeGxncFk/ view>. Acesso em: 20/03/2015.

KELLER, N.P.; BERGSTROM, G.C. Developmental predisposition of maize to anthracnose stalk rot. Plant Disease, v.72, p.977-980, 1988. Disponível em: <http://www.apsnet.org/publications/PlantDisease/ BackIssues/Documents/1988Articles/PlantDisease72n11_977.PDF>. Acesso em: 20/03/2015. doi: 10.1094/PD-72-0977.

LEITE, R.M.V.B.C.; AMORIM, L. Elaboração e validação de escala diagramática para Mancha de Alternária em girassol. Summa 
Phytopathologica, v.28, p.14-19, 2002. Disponível em: <https:/ docs.google.com/file/d/0B6r87-taaOV7R0h1cF9kcmhsaW8/ view>. Acesso em: 20/03/2015.

MATIELLO, R.R. et al. Comparison of yield damage of tropical maize hybrids caused by anthracnose stalk rot. Tropical Plant Pathology, v.38, p.128-132, 2013. Disponível em: $<$ http://www. scielo.br/pdf/tpp/v38n2/a06v38n2.pdf>. Acesso em: 20/03/2015. doi: 10.1590/S1982-56762013000200006.

MICHEREFF, S.J. et al. Escala diagramática e tamanho de amostras para avaliação da severidade da mancha parda da mandioca (Cercosporidium henningsii). Agrotrópica, v.10, p.143-148, 1998. Disponível em: <http://www.ceplac.gov.br/ paginas/agrotropica/>. Acesso em: 20/03/2015.

MICHEREFF, S.J. et al. Development and validation of a diagrammatic key for Cercospora leaf spot of sweet pepper. Summa Phytopathologica, v.32, p.260-266, 2006. Disponível em: <http://www.scielo.br/pdf/sp/v32n3/a08v32n3.pdf>. Acesso em: 20/03/2015. doi: 10.1590/S0100-54052006000300008.

MUIMBA-KANKOLONGO, A.; BERGSTROM, G.C. Wound predisposition of maize to anthracnose stalk rot as affected by internode position and inoculum concentration of Colletotrichum graminicola. Plant Disease, v.76, p.188-195, 1992. Disponível em: $<$ http://www.apsnet.org/publications/PlantDisease/BackIssues/ Documents/1992Articles/PlantDisease76n02_188.PDF>. Acesso em: 20/03/2015. doi: 10.1094/PD-76-0188.

MUIMBA-KANKOLONGO, A; BERGSTROM, G.C. Reduced anthracnose stalk rot in resistant maize is associated with restricted development of Colletotrichum graminicola in pith tissues. Journal of Phytopathology, v.159, p.329-341, 2011. Disponível em: <http://onlinelibrary.wiley.com/doi/10.1111/ j.1439-0434.2010.01766.x/epdf>. Acesso em: 20/03/2015. doi: 10.1111/j.1439-0434.2010.01766.x.

NEWTON, A.C.; HACKETT, C.A. Subjective components of mildew assessment on spring barley. European Journal of Plant Pathology, v.100, p.395-412, 1994 Disponível em: <http://download.springer.com/static/pdf/323/ art \%253A10.1007\%252FBF01874807.pdf?auth66=1426886374 43a1f98f764ecf2a2096bc9b2e7ade2e\&ext=.pdf>. Acesso em: 20/03/2015. doi: 10.1007/BF01874807.

NITA, M. et al. Reliability and accuracy of visual estimation of Phomopsis leaf blight of strawberry. Phytopathology, v.93, p.995-1005, 2003. Disponível em: <http://apsjournals.apsnet.org/ doi/pdf/10.1094/PHYTO.2003.93.8.995>. Acesso em: 20/03/2015. doi: 10.1094/PHYTO.2003.93.8.995.
NUTTER, F.W.; SCHULTZ, P.M. Improving the accuracy and precision of disease assessments: selection of methods and use of computer-aided training programs. Canadian Journal of Plant Pathology, v.17, p.174-184, 1995. Disponível em: <http://www. tandfonline.com/doi/pdf/10.1080/07060669509500709>. Acesso em: 20/03/2015. doi: 10.1080/07060669509500709.

PARKER, S.R. et al. The reliability of visual estimates of disease severity on cereal leaves. Plant Pathology, v.43, p.856865, 1995. Disponível em: <http://onlinelibrary.wiley.com/ doi/10.1111/j.1365-3059.1995.tb02745.x/epdf $>$. Acesso em: 20/03/2015. doi: 10.1111/j.1365-3059.1995.tb02745.x.

RIOS, J.A. et al. Development and validation of a standard area diagram set to assess blast severity on wheat leaves. European Journal of Plant Pathology, v.136, p.603-611, 2013. Disponível em: <http://download.springer.com/static/pdf/573/ art\%253A10.1007\%252Fs10658-013-0191-x.pdf?auth66=14268 87166_6eaef9af910976f048dc275fec70a864\&ext=.pdf>. Acesso em: 20/03/2015. doi: 10.1007/s10658-013-0191-x.

STONEHOUSE, J. Assessment of Andean bean diseases using visual keys. Plant Pathology, v.43, p.519-527, 1994. Disponível em: $<$ http://onlinelibrary.wiley.com/doi/10.1111/j.1365-3059.1994. tb01586.x/epdf $>$. Acesso em: 20/03/2015. doi: 10.1111/j.13653059.1994.tb01586.x.

TODD, L.R.; KOMMEDAHL, T. Image analysis and visual estimates for evaluating disease reactions of corn to Fusarium stalk rot. Plant Disease, v.78, p.876-878, 1994. Disponível em: $<$ https://www.apsnet.org/publications/PlantDisease/BackIssues/ Documents/1994Articles/PlantDisease78n09_876.PDF>. Acesso em: 20/03/2015. doi: 10.1094/PD-78-0876.

VENARD, C; VAILLANCOURT, L. Colonization of fiber cells by Colletotrichum graminicola in wounded maize stalks. Phytopathology, v.97, p.438-447, 2007. Disponível em: $<$ http:// apsjournals.apsnet.org/doi/pdf/10.1094/PHYTO-97-4-0438>. Acesso em: 20/03/2015. doi: 10.1094/PHYTO-97-4-0438.

YADAV, N.V. et al. Development and validation of standard area diagrams to aid assessment of pecan scab symptoms on fruit. Plant Pathology,v.61,p.325-335,2012. Disponívelem: $<$ http://onlinelibrary. wiley.com/doi/10.1111/j.1365-3059.2012.02641.x/epdf $>$. Acesso em: 20/03/2015. doi: 10.1111/j.1365-3059.2012.02641.x.

YI, Q. et al. Reliability analysis for continuous measurements: equivalence test for agreement. Statistics in Medicine, v.27, p.2816-2825, 2008. Disponível em: <http://onlinelibrary.wiley. com/doi/10.1002/sim.3110/epdf $>$. Acesso em: 20/03/2015. doi: $10.1002 / \operatorname{sim} .3110$ 